\title{
Research on glass processing under manufacturing cutting machine fault prediction in cloud manufacturing
}

\author{
Hongxia Cai ${ }^{1, a,}$, Zhuangyu Wei ${ }^{2, b, ~ * ~ a n d ~ M i n s h a n ~ R e n ~}{ }^{2, c}$ \\ ${ }^{1}$ School of Mechatronic Engineering and Automation, Shanghai Key Laboratory of Intelligent Manufacturing \\ and Robotics, Shanghai University \\ ${ }^{2}$ Graduate Institute of Mechatronic Engineering and Automation, Shanghai University

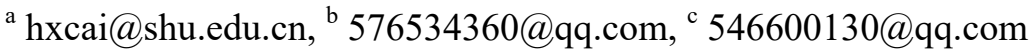 \\ * Zhuangyu Wei
}

Keywords: Cutting machine; Fault prediction; Cloud manufacturing; Aproiri; Association rules; Data mining.

\begin{abstract}
The prediction of equipment malfunction is crucial to guarantee its safe operation and improve its management efficiency. Combining with the character of cloud manufacturing, this paper makes the malfunction prediction of the cutting machine come true, which is based on the improved Apriori algorithm. First, with the malfunction analysis of the cutting machine, we can set up the comparison table of malfunction features and that of parameter, integrate different manufacturer and data of different equipment into virtual cloud pool and store them in HDFS form. Then, we can explore the association rules of the machine malfunction data when we use Hadoop parallelisation to improve Apriori algorithm and add the temporal concepts. So that we can predict the trend of cutting machine malfunction and support the subsequent health management.
\end{abstract}

\section{Introduction}

Nowadays, there are thousands of kinds of processed glass in the world and its product scale of systematization, standardization and energy efficiency has been realized [1]. With the expansion of scale, the cutting machine has played an important role in automation and their users demand on higher safety and reliability, besides, the malfunction rate is increasing remarkably, high speed automatic and complex cutting machine proposes urgent requirement on its reliability [2]. The traditional glass processing industries cannot troubleshoot the malfunction, they mainly depend on manual maintenance regularly, and therefore they may suffer from the challenge of adapting to the market environment and improving the ability of innovation [3]. To the cutting machine, its complex construction makes it hard to set a physical model of characterization or system degradation and residual life, and with the increase of business system, the rise trend of data is explosive, the daily data can be up to over ten TB, so the efficiency of malfunction prediction based on the traditional data model becomes lower [4].

Cloud computing serves as a new type of cloud information technology, it provides customers with software and hardware service in the self-help mode through network [5]. Its expandability, environmental sharing and flexibility provide the enterprise with flexible, low-cost and controllable information technology solutions. Association rules mining serves as one of the most mature and the most active research contents, its target is to find out the correlation between different items and its core problem is the acquisition of the frequent item sets. As a core algorithm, Apriori algorithm can get all frequent item sets through scanning the database several times, and under the condition of mass data, the scanning of the database will waste a large amount of time and memory space [6]. Therefore, using the parallel thinking to improve will become a hot spot of research this year; cloud computing support parallel improving with its unique advantage.

This paper uses Hadoop cloud computing to parallel and improve the traditional Apriori algorithm to predict malfunction, and it focuses on extracting the malfunction features of the cutting 
machine and realizing of Map/Reduce in Apriori algorithm.

\section{Fault prediction analysis of cutting machine}

The cutting machine in the glass processing factory experiences long-term high-impact vibration and shock with machinery problems and electrical problems, which result in the occurrence of machine malfunction and badly influence the normal process of the glass and bring the glass industries a series of great loss. How to early warn with the machine malfunction and control the parts or elements expecting to appear in advance, and avoid the loss from the malfunction is a significant matter. We can collect the common faults, make sure the characterization and parameter that can feature malfunction and set the malfunction data model.

\subsection{Components of the glass cutting machine}

Glass cutting machine consists of 3 parts: cutting table, cutting bridge and computer control box [7].

1.cutting table: it is made up of bracket, desktop, conveyor, and driving devices and air cushion system and so on.

2.Cutting bridge: it is a metal bridge stretching over the cutting table, it supports longitudinal metal guide rail.

3.Computer control box: it is the operating cabinet of automation in centralized installation, and its hardware schematics are as follow.
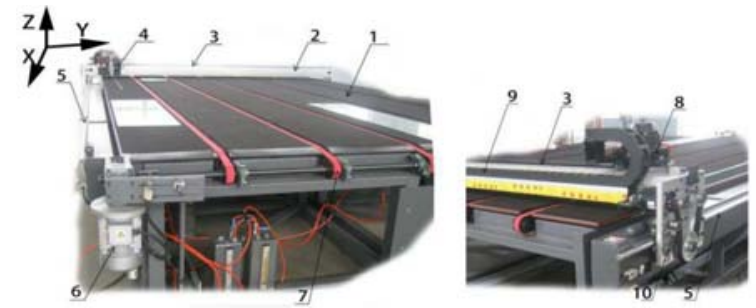
1 Wool felt gasket
2 Beam
3 Horizontal guide rail
4 Cutter head
5 Vertical guide rail
6 Drive motor 7 Conveyor belt
8 Lateral pass delivery system
9 Cable track
10 Longitudinal cutter transmission system

Figure 1 Glass Cutting System

\subsection{Operational principle of the glass cutting system}

The glass cutting system of the glass cutting numerical control system mainly consists of NC control system leaded by Trio motion controller, glass cutting machine, upper sheet mechanism, snapping mechanism and rack.

The table of working relationship among glass cutting NC numerical control system, glass cutting mechanism, upper sheet mechanism, snapping mechanism is demonstrated in picture.

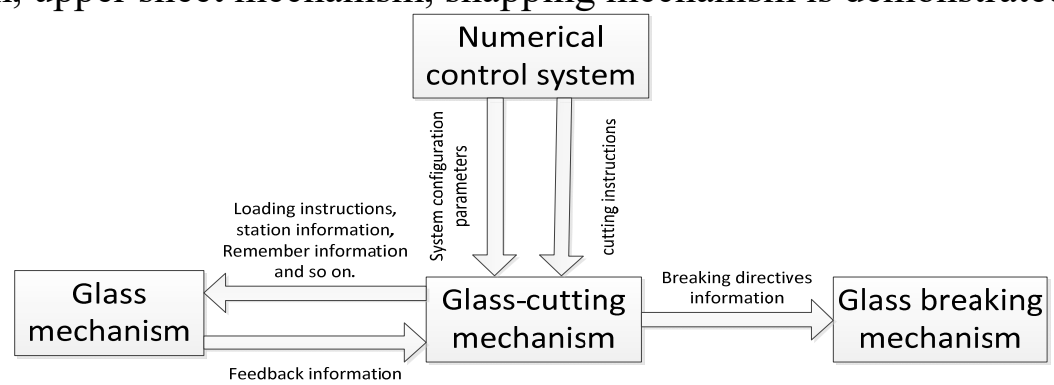

Figure 2 Glass cutting and CNC system schematic diagram of mechanism

$\mathrm{NC}$ numerical control system sends orders to control the cutting machine; while the cutting machine sends the information of possession of glass, legitimacy of glass, triggering of the limit switch, etc. to the upper sheet mechanism or the snapping mechanism; when the upper sheet mechanism achieves the signal, it will offer the raw glass sheet to the cutting machine. After finishing cutting, glass cutting mechanism will send the signal to the snapping mechanism, whose 
function is to save the time of manual work and complete it automatically [8].

Through analyzing the components and operation principle of the cutting machine, we can know the cutting equipment is a system combining machine, electricity, liquid gas. When we use Apriori algorithm to predict the malfunction, we can find out the association rules of malfunction features and parameters on the basis of existing malfunction, so that we can provide help for decision maintenance. With the recorded data and the analysis of the principle of machine, we arrange the performance of the cutting malfunction. We can mark the abnormal data with fault code. As the following table 1:

Table 1 The abnormal data with fault code

\begin{tabular}{|c|c|c|c|}
\hline Fault logging & $\begin{array}{l}\text { Fault } \\
\text { code }\end{array}$ & Fault parameter performance & $\begin{array}{l}\text { Fault } \\
\text { codes }\end{array}$ \\
\hline Engine fault & $\mathrm{F} 1$ & Engine speed limit or below the lower limit & F10 \\
\hline $\begin{array}{c}\text { Cooling liquid temperature } \\
\text { anomaly }\end{array}$ & F2 & $\begin{array}{c}\text { Coolant temperature exceeds the upper limit or } \\
\text { below the lower limit }\end{array}$ & F11 \\
\hline Gear fault & F3 & Cutting the speed limit or below the lower limit & F12 \\
\hline Control board failure & F4 & $\begin{array}{l}\text { Cutting pressure exceeds the upper limit or below } \\
\text { the lower limit }\end{array}$ & F13 \\
\hline On-chip fault & F5 & $\begin{array}{c}\text { Pressure exceeds upper limit or below the lower } \\
\text { limit }\end{array}$ & F14 \\
\hline Linearity deviant fault & F6 & $\begin{array}{l}\text { Angle wheel exceeds upper limit or below the } \\
\text { lower limit }\end{array}$ & F15 \\
\hline $\begin{array}{l}\text { Cutting glass fragments } \\
\text { fault }\end{array}$ & F7 & The cutting pressure exceeds upper limit & F16 \\
\hline Hydraulic system fault & F8 & $\begin{array}{c}\text { Hydraulic pressure exceeds the upper limit or } \\
\text { below the lower limit }\end{array}$ & F13 \\
\hline Breaking fault & F9 & Breaking too early or too late & F17 \\
\hline
\end{tabular}

\section{Malfunction data model}

When the traditional Apriori algorithm scans the database of mass storage, it will waste a large amount of time and memory space; while we use Apriori traditional association rules algorithm, we can divide the data from the malfunction event into sectors with equipment code and record the time when malfunction appears, ruling out the invalid data, so that we can get the temporal malfunction data model. Taking Map/Reduce, we can conserve the algorithm spreading on different nodes in order to highly parallel, which can guarantee the accuracy of the prediction and improve the operation efficiency. The malfunction data model is as the chart shows.

Table 2 Sequential model of fault data

\begin{tabular}{|c|c|c|c|c|c|}
\hline Transaction(Device number) & \multicolumn{5}{|c|}{ Project I (Fault Data) } \\
\hline M1 & $<$ R1,Ta1 $>$ & $<$ R2,Ta2 $>$ & $<$ R3,Ta3 $>$ & $<$ R2,Ta4 $>$ & $<$ R5,Ta5 $>$ \\
\hline M2 & $<$ R2,Tb1 $>$ & $<$ R3,Tb2 $>$ & $<$ R4,Tb3 $>$ & $<$ R5,Tb4 $>$ & $<$ R3,Tb5 $>$ \\
\hline M3 & $<$ R1,Tc1 $>$ & $<$ R3,Tc2 $>$ & $<$ R4,Tc3 $>$ & $<$ R5,Tc4 $>$ & $<$ R2,Tc5 $>$ \\
\hline M4 & $<$ R1,Td1 $>$ & $<$ R2,Td2 $>$ & $<$ R3,Td3 $>$ & $<$ R4,Td4 $>$ & $<$ R5,Td5 $>$ \\
\hline M5 & $<$ R4,Te1 $>$ & $<$ R1,Te2 $>$ & $<$ R5,Te3 $>$ & $<$ R2,Te4 $>$ & $<$ R1,Te5 $>$ \\
\hline M6 & $<$ R2,Tf1 $>$ & $<$ R1,Tf2 $>$ & $<$ R4,Tf3 $>$ & $<$ R5,Tf4 $>$ & $<$ R3,Tf5 $>$ \\
\hline
\end{tabular}

The data construction of the model needs to satisfy the two characters:

Character one: each data has only one equipment code, all the data items correspond to their equipment malfunction data.

Character two: all the data items in the data collections are described with two- tuples $<\mathrm{i}, \mathrm{t}>$, which is called temporal item, and i means malfunction, $t$ represents time halberd of the temporal items.

\subsection{Equipment data integration under cloud manufacturing}

Malfunction features and abnormal parameters have different ways of acquisition, and mass malfunction feature data is conserved in enterprises, this kind of data can be live transmitted 
without collecting device, instead, it can be shared by sharing database or in the way of files and WebService. While we can adopt a box equipment to acquire the abnormal parameters.

With equipment manufactures, system integrator and the operational guarantee of the industries, it features with real-time operation guarantee, communication of networks and equipment, stable data transmission, real-time monitoring, and remotely warning. Its specific implementation procedures are as follows: first, box can live control the inner part of enterprise, integrate data with net transmission, and with the unified data format specifications, the abnormal parameters can be uploaded to the inner system of the enterprise, then the different system will transmit the data to the traditional database Oracle, then different enterprises deliver data through WebService to Cloud with conservation of HDFS form. The use of the box solves the problem that coding the equipment information from different systems.

\subsection{Improved Apriori algorithm basic principle and steps}

Apriori algorithm is usually used to mine Boolean algorithm, single layer, one dimensional association. Its core idea is the idea of two-phase frequent sets based on recurrence. Apriori algorithm takes the priority knowledge of the frequent item sets, which takes the iteration form of layer-by-layer search, and we can search $\mathrm{k}+1$ item set through item $\mathrm{k}$ [9].

Map/Reduce is a distributed computation model that can be used for mass data processing, and it can organize cluster to process large data set, is a main parallel data process model on the cloud computing platform [10]. In order to make sure that the result is more persuasive, this paper introduce the temporal concept. Defining operator “\#”, expression\# (i, T), if T is Maximum value of ordered item, its outcome is 1 , vice verse, its outcome is 0 . Temporal rules subtraction is as flows:

$$
(\mathrm{A}, \mathrm{T} \alpha)-(\mathrm{B}, \mathrm{T} \beta)=\mathrm{A} \rightarrow \mathrm{B}
$$

The generating process of the frequent item sets is as follows:

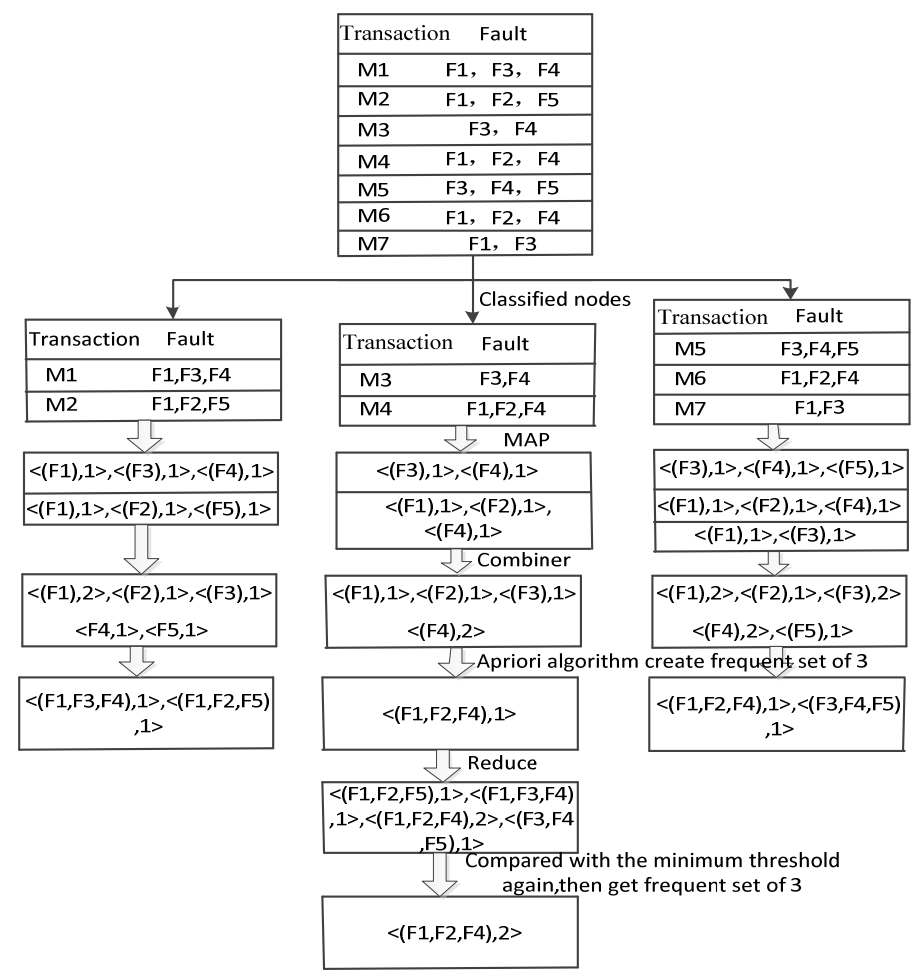

Figure 2 Calculation flowchart

The generating process of the rules is as follows:

a) we can use the form of HDFS to output the result to the files, and segment each line of the files. To form $<$ key 1 , value $1>$, key 1 represents line offset valuel represents one frequent item, Map function is used to scan key value pair and call generating function, and output $<$ frequent item, rule $>$ form.

$<$ frequent item, rule $>$ can be written as: $<(\mathrm{Fi}, \mathrm{Fj}, \mathrm{Fk}),(\mathrm{Fi}, \mathrm{Fj})=\mathrm{Fk}$, support, reliability, $\Delta \mathrm{t}>$ 
$\Delta \mathrm{t}$ can be gotten by using the time pattern in minus. For example, $(\mathrm{Fi}, \mathrm{Fj}), \mathrm{T} 1)-(\mathrm{Fk}, \mathrm{T} 2)=(\mathrm{F} 1, \mathrm{~F} 2)=\mathrm{F} 4, \Delta \mathrm{T}=\mathrm{T} 2-\mathrm{T} 1$

When $\Delta \mathrm{T}<0$, we can quit it. And in this example, we can get the result through Map function:

$<(\mathrm{F} 1, \mathrm{~F} 2, \mathrm{~F} 4),(\mathrm{F} 1, \mathrm{~F} 2)=\mathrm{F} 4,0.2,67 \%, \Delta \mathrm{T} 1>$ means the degree of support is 0.2 , and the degree of reliance is $67 \%$;

$<(\mathrm{F} 1, \mathrm{~F} 2, \mathrm{~F} 4),(\mathrm{F} 1, \mathrm{~F} 4)=\mathrm{F} 2,0.2,67 \%, \Delta \mathrm{T} 2>$ means the degree of support is 0.2 and the degree of reliance is $67 \%$;

b) Reduce can get results through specification: $<(\mathrm{F} 2, \mathrm{~F} 4)=\mathrm{F} 1,100 \%: \Delta \mathrm{T} 3$ ', $>$, which can be described as: this kind of equipment appear at malfunction $\mathrm{F} 2$ and $\mathrm{F} 4$, and the next $\Delta \mathrm{T} 3$ will meet the malfunction $\mathrm{F}$ 1, and the final result will be conserved in HDFS.

Through Map/Reduce paralyzing, we only need to scan D twice to mine all frequent item sets, which cut back on a lot on the algorithm expenses. And the mining process of the frequent item sets in each node is independent, which can lower the communication of the nodes, promote the efficiency of the algorithm; adding the temporal concept will make the prediction more accurate.

\section{Example verification}

To illustrate with examples, combining with MapReduce, this Hadoop distributed file system HDFS that applied in this study can bring higher throughout of data access, and its application takes the form of WORM (write once, read multiple). The table below shows some temporal malfunction data in glass processing of some enterprise.

Table 3 Sequential model of fault data

\begin{tabular}{|c|c|c|c|c|c|c|c|}
\hline \multicolumn{2}{|c|}{$\begin{array}{c}\text { Device } \\
\text { Number }\end{array}$} & $\begin{array}{c}\text { GSQ6033A2P } \\
0000001\end{array}$ & $\begin{array}{c}\text { GSQ6033A2P } \\
0000002\end{array}$ & $\begin{array}{c}\text { GSQ6033A2P } \\
0000003\end{array}$ & $\begin{array}{c}\text { GSQ6033A2 } \\
\text { P0000004 }\end{array}$ & $\ldots$ & $\begin{array}{c}\text { GSQ6033A2P0 } \\
001000\end{array}$ \\
\hline \multirow{17}{*}{$\begin{array}{l}\text { Fault } \\
\text { Code }\end{array}$} & 1 & $(1,34)$ & $(0,0)$ & $(1,58)$ & $(1,44)$ & $\ldots$ & $(0,0)$ \\
\hline & 2 & $(0,0)$ & $(1,115)$ & $(0,0)$ & $(1,77)$ & $\ldots$ & $(1,19)$ \\
\hline & 3 & $(1,121)$ & $(1,39)$ & $(0,0)$ & $(0,0)$ & $\ldots$ & $(1,26)$ \\
\hline & 4 & $(1,47)$ & $(1,67)$ & $(1,120)$ & $(1,71)$ & $\ldots$ & $(0,0)$ \\
\hline & 5 & $(0,0)$ & $(0,0)$ & $(1,29)$ & $(1,48)$ & $\ldots$ & $(0,0)$ \\
\hline & 6 & $(1,29)$ & $(1,31)$ & $(0,0)$ & $(1,51)$ & $\ldots$ & $(1,54)$ \\
\hline & 7 & $(1,79)$ & $(1,81)$ & $(0,0)$ & $(1,25)$ & $\ldots$ & $(0,0)$ \\
\hline & 8 & $(1,46)$ & $(1,135)$ & $(1,50)$ & $(0,0)$ & $\ldots$ & $(1,17)$ \\
\hline & 9 & $(0,0)$ & $(0,0)$ & $(1,38)$ & $(1,84)$ & $\ldots$ & $(1,57)$ \\
\hline & 10 & $(1,38)$ & $(1,23)$ & $(0,0)$ & $(1,43)$ & $\ldots$ & $(1,33)$ \\
\hline & 11 & $(0,0)$ & $(1,121)$ & $(0,0)$ & $(1,36)$ & $\ldots$ & $(1,64)$ \\
\hline & 12 & $(1,52)$ & $(1,34)$ & $(1,41)$ & $(0,0)$ & $\ldots$ & $(0,0)$ \\
\hline & 13 & $(1,69)$ & $(0,0)$ & $(1,15)$ & $(0,0)$ & $\ldots$ & $(1,24)$ \\
\hline & 14 & $(0,0)$ & $(1,28)$ & $(0,0)$ & $(1,56)$ & $\ldots$ & $\frac{(1, L 1)}{(0,0)}$ \\
\hline & 15 & $(1,48)$ & $(1,39)$ & $(0,0)$ & $(0,0)$ & $\ldots$ & $(1,36)$ \\
\hline & 16 & $(1,47)$ & $(1,38)$ & $(1,40)$ & $(0,0)$ & $\ldots$ & $(1,12)$ \\
\hline & 17 & $(1,31)$ & $(0,0)$ & $(1,52)$ & $(1,65)$ & $\ldots$ & $(0,0)$ \\
\hline
\end{tabular}

Supposing the minimum support threshold is 4 , that is, min-sup $=0.23, \mathrm{M}=5$ (database is divided into 5 parts), $\mathrm{R}=1$ (allocate one Reduce task).

the rule that conforms to the criteria is acquired through Map function:

Supposing $\min$-conf $=75 \%$.

Table 4 Frequent set of four

\begin{tabular}{|c|c|}
\hline Frequent item sets & Rules \\
\hline (F10,F12,F13) $\Rightarrow \mathrm{F} 1$ & $<(\mathrm{F} 10, \mathrm{~F} 12, \mathrm{~F} 13) \Rightarrow \mathrm{F} 1,85 \%: 14 ">$ \\
\hline$(\mathrm{F} 2, \mathrm{~F} 3, \mathrm{~F} 12) \Rightarrow \mathrm{F} 3$ & $<(\mathrm{F} 2, \mathrm{~F} 3, \mathrm{~F} 11) \Rightarrow \mathrm{F} 3,90 \%: 10 ">$ \\
\hline$(\mathrm{F} 15, \mathrm{~F} 14, \mathrm{~F} 10) \Rightarrow \mathrm{F} 6$ & $<(\mathrm{F} 10, \mathrm{~F} 14, \mathrm{~F} 15) \Rightarrow \mathrm{F} 6,88 \%: 15 ">$ \\
\hline$(\mathrm{F} 7, \mathrm{~F} 16, \mathrm{~F} 17) \Rightarrow \mathrm{F} 9$ & $<(\mathrm{F} 7, \mathrm{~F} 16, \mathrm{~F} 17) \Rightarrow \mathrm{F} 9,100 \%: 6 ">$ \\
\hline$(\mathrm{F} 12, \mathrm{~F} 13, \mathrm{~F} 14) \Rightarrow \mathrm{F} 8$ & $<(\mathrm{F} 12, \mathrm{~F} 14, \mathrm{~F} 13) \Rightarrow \mathrm{F} 8,92 \%: 9 ">$ \\
\hline
\end{tabular}

With the arithmetic of Reduce, we can describe the result, such as $<(\mathrm{F} 10, \mathrm{~F} 12, \mathrm{~F} 13)=\mathrm{F} 1$, $85 \%: 14>$, this kind of equipment appear after the malfunction number F10, F12, F13, and after the next 14 days, the F1 will be abnormal, so the cycle of malfunction is 14 days, and the output will be 
delivered to HDFS.

\section{Conclusion}

The prediction of equipment malfunction is a crucial method for improving its stability and reliability. However, with the variety of requirements of enterprises and the exponential increase of data, the research under cloud manufacturing is still less than expected. This paper will take the features of the cutting machine in the deep processing of the glass into account, introduce the open source technology Map/Reduce that applies to big data analysis, and optimize the traditional Apriori algorithm, and verify with examples that the above method has faster arithmetic speed and more accurate association rules mining. In addition, this paper is highly adaptable and has a good application prospect and promotion value in solving complex equipment malfunction prediction. However, Hadoop technology and its related technology demand highly on the implementer and the paper does not refer to the equipment maintenance and so that the incident that result in the equipment malfunction is still not appropriate. Therefore, the further target is to focus on some easy parallel data processing and further study on the factors that cause malfunction of the cutting machine.

\section{Acknowledgements}

This work was supported by Shanghai Economic and Information Commission under Grant No. Shanghai CXY2016-007. We are grateful for the financial support, and also would like to thank the anonymous reviewers and the editor for their comments and suggestions.

\section{References}

[1] $\mathrm{Yu}$ Lin. The glass industry to technological innovation and optimize the structure of the track. China Building Materials News, 2011(1):1-2.

[2] Renwei Zhang. Transforming the pattern of economic development in taking the roadof sustainable development. Architectural glass and technical gall, 2011(6):87-92.

[3] Shenliang Gu, Shenchang Chen. Foreign flat glass and glass-processing technology. Henan building materials, 2003(1):8-9.

[4] Yang Wang, International flat glass and glass deep processing industry. Building materials industry, 2002(4):8-9.

[5] Morrissey Matt. Industry set for Beijing as China glass approaches. Glass International. 2010,5,33(1):37-38.

[6] Zhipeng Hao, Shengkui Zeng, Jianbing Guo, etc. A quantitative model for the reliability of knowledge and data fusion. Journal of Beijing University,2015, 35(10):18-27.

[7] CAESRENDRA W, WIDODO A, YANG B S. Combination of Probability Approach and Support Vector Machine Towards Machine Health Prognostics. Probabilistic Engineering Mechanics,2011,26(26):165-173.

[8] AGRAEAL R, IMIELINSKIT, SWAMI A. Mining Sociation Rules between Sets of Items in Large Databases. Washington DC, United states, 1993:207-216.

[9] Li Y, Kurfess T R, Liang Y. Stochastic Prognostics for Rolling Element Bearings. Mechanical Systems and Signal Processing, 2000, 14(5): 747-762.

[10] Ying Peng, Ming Dong. A hybrid approach of HMM and grey model for age-dependent health prediction of engineering assets. Expert Systems with Applications, 2011, 38: 12946-12953. 